\title{
The structure of small intestinal tissue in pigs as influenced by indigestible polysaccharides*
}

\author{
A. Tuśnio', E. Święch, M. Taciak and L. Buraczewska \\ The Kielanowski Institute of Animal Physiology and Nutrition, \\ Polish Academy of Sciences \\ 05-110 Jabłonna, Poland
}

\begin{abstract}
The experiment was carried out to investigate the effect of non-starch polysaccharides on the small intestinal morphology of pigs. Growing pigs were fed for 40 days on one of four diets differing in the level and type of polysaccharide. They received the control diet with or without 4 or $8 \%$ pectin or a diet with rye instead of part of the wheat. The influence of polysaccharides on the epithelial structure was evaluated in the duodenum, mid-jejunum and ileum. Increased crypt depth and mucosa thickness were found after feeding the diet with rye in all measured segments, whereas feeding the diets with 4 and $8 \%$ pectin caused an increase in myenteron thickness of the mid-jejunum and ileum, in comparison with other diets.
\end{abstract}

KEY WORDS: pig, non-starch polysaccharides, small intestine, morphology

\section{INTRODUCTION}

The impact of the diet on the development of the gastrointestinal (GI) tract of growing animals has become of particular concern in nutrition research. The small intestine is the most important segment of the GI tract as this is where digestion and absorption of the majority of nutrients take place. The polysaccharides not digested in this part of the GI tract can influence the structure of the small intestine by alteration of its length and weight (Brunsgaard et al., 1995; van der Klis and Jansman, 2002). These effects are often associated with modification of the gut epithelium morphology and, consequently, of the hydrolytic and absorptive functions of the epithelium (Montagne et al., 2003).

The purpose of this study was to determine the effect of different levels and sources of non-starch polysaccharides (NSP) on the morphology of the small intestine of growing pigs.

\footnotetext{
* Supported by the State Committee for Scientific Research, Grant No. 2P06Z06827

${ }^{1}$ Corresponding author: e-mail: a.tusnio@ifzz.pan.pl
} 


\section{MATERIAL AND METHODS}

The experiment was approved by the Local Animal Care Commission. Twenty four male pigs with a high lean gain potential (synthetic line 990, Poland), divided into four groups consisting of six pigs each and of an initial body weight of about $20 \mathrm{~kg}$, were used in the study. The animals were kept in metabolic cages in an environmentally controlled room $\left(22^{\circ} \mathrm{C}\right)$ with ad libitum access to water. The main ingredients of the diets were wheat, maize, soyabean meals, casein and crystalline amino acids. The diets were supplemented with 0,4 or $8 \%$ highly esterified apple pectin (Pectowin, Poland); the fourth diet contained rye instead of part of the wheat. The diets were balanced (about 18\% protein) and each diet was fed for 40 days. After this time the animals were sacrificed and the small intestine was dissected into the duodenum, mid-jejunum and ileum and the weight and length of each part were recorded. Samples of tissue from each segment were taken for histological analysis and placed in Bouin's solution. Sections were cut and stained with haematoxylin and eosin. Villus height, crypt depth, thickness of the tunica mucosa and myenteron were determined. Data were analysed using the One-way ANOVA procedure and mean values were compared by Duncan's test. The calculations were carried out using the STATGRAPHICS Plus ver. 3.0 package.

\section{RESULTS}

Supplementation of NSP in the diet did not alter the weight and the length of different parts of the small intestine. The results of histometry are shown in Figure 1. The intensity and type of modification of the small intestine tissue induced by pectin and rye differed. Feeding the diet with rye increased crypt depth and mucosa thickness in all measured segments of the small intestine. It also increased $(\mathrm{P} \leq 0.05)$ the length of villi in the mid-jejunum, but did not change the size of the myenteron compared with the other diets. Feeding the diet containing $4 \%$ pectin reduced $(\mathrm{P} \leq 0.05)$ the length of villi and increased myenteron thickness in the duodenum. Both pectin diets significantly reduced crypt depth in the midjejunum and enlarged myenteron thickness in the mid-jejunum $(\mathrm{P} \leq 0.05)$ and ileum as compared with the other diets.

\section{DISCUSSION}

The dietary fibre of rye belongs to cell-wall NSP (mixed linked $\beta$-glucans, arabinoxylans) and to non-cell-wall NSP (fructans). Apple pectin is a fraction of non-cell-wall NSP. The effect of these fractions of dietary fibre on intestinal 

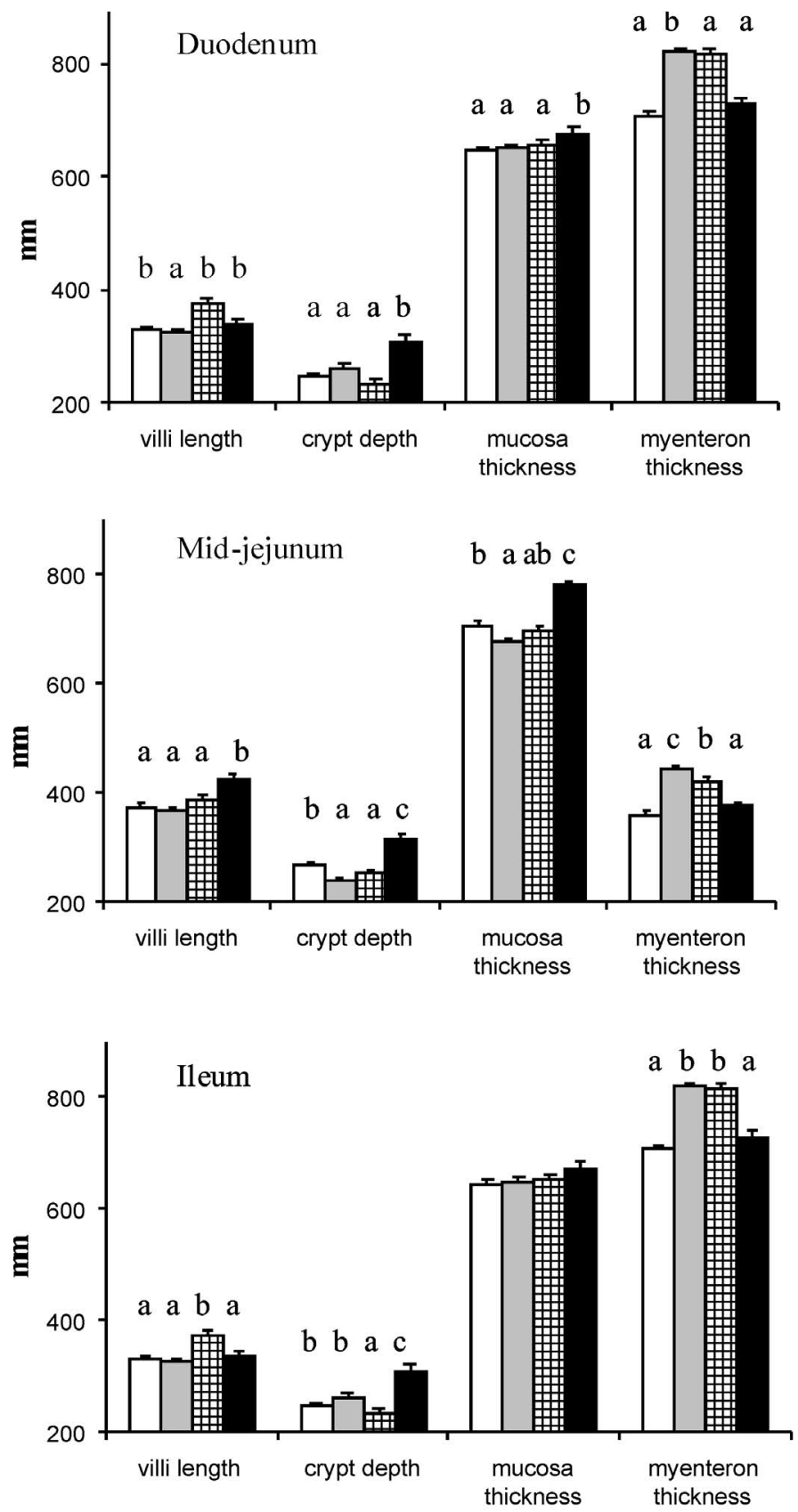

Figure 1 . The effect of feeding diets with $0 \%$ pectin $(\square), 4 \%$ pectin $(\square), 8 \%$ pectin (团) and diet with rye $\square$ on the histological parameters of the duodenum, mid-jejunum and ileum of growing pigs (mean+SE; a,b,c $\mathrm{P} \leq 0.05$ ) 
epithelial anatomy depends on their ability to increase digesta viscosity. According to Montagne et al. (2003), high viscosity digesta may increase the rate of villus cell losses, leading to villus atrophy, a phenomenon associated with increased crypt-cell production, and generally with increased crypt depth. The results of our experiment do not confirm these observations, since there were no differences in the length and weight of the small intestine or reduction of villus length in the experimental groups as compared with the control diet (standard). Only feeding the diet with rye caused increased crypt depth in all measured segments of the small intestine. In contrast with the rye diet, diets with two levels of pectin decreased crypt depth in the mid-jejunum. The increase of crypt-cell proliferation, resulting in a greater crypt depth can be explained by the trophic effect of short-chain fatty acids, the main products of bacterial NSP metabolism in the gastrointestinal tract. This mechanism is still incompletely understood. In experiments on germ-free rats (Sakata and Inagaki, 2001) it was confirmed that the influence of short-chain fatty acids is not restricted to the colon but they also can stimulate epithelial cell growth of the small intestine.

\section{CONCLUSIONS}

Non-starch polysaccharides affected the morphology of the small intestine, however, the mechanism of their action requires further investigation.

\section{REFERENCES}

Brunsgaard G., Eggum B.O., Sandström B., 1995. Gastrointestinal growth in rats as influenced by indigestible polysaccharides and adaptation period. Comp. Biochem. Physiol. 111, 369-377

Montagne L., Pluske J.R., Hampson D.J., 2003. A review of interactions between dietary fibre and the intestinal mucosa, and their consequences on digestive health in young non-ruminant animals. Anim. Feed Sci. Tech. 108, 95-117

Sakata T., Inagaki A., 2001. Organic acid production in the large intestine: implication for epithelial cell proliferation and cell death. In: A. Piva, K.E. Bach Knudsen, J.E. Linderberg (Editors). Gut Environment of Pigs. Nottingham University Press, pp. 85-94

Van der Klis J.D., Jansman A.J.M., 2002. Optimising nutrient digestion, absorption and gut barrier function in monogastrics: Reality or illusion? In: M.C. Blok, H.A. Vahl, L. de Lange, A.E. van de Braak, G. Hemke, M. Hessing (Editors). Nutrition and Health of the Gastrointestinal Tract. Wageningen Academic Publishers, pp. 15-36 